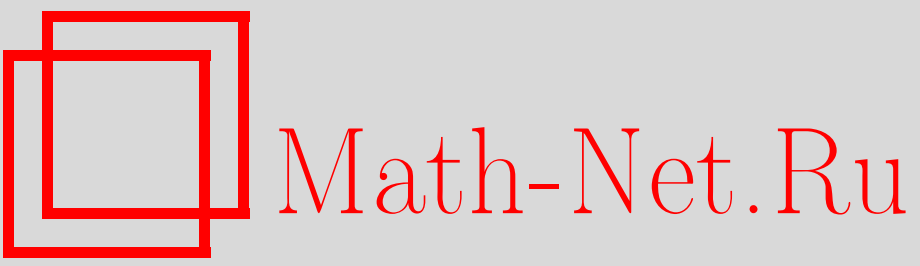

Л. Рыпарова, Й. Микеш, Отображения и преобразования поворота, Итоги науки и техн. Сер. Соврем. мат. и ее прил. Темат. обз., 2020, том 180, 91-95

DOI: https://doi.org/10.36535/0233-6723-2020-180-91-95

Использование Общероссийского математического портала Math-Net.Ru подразумевает, что вы прочитали и согласны с пользовательским соглашением http: //www.mathnet.ru/rus/agreement

Параметры загрузки:

IP: 35.173 .219 .149

26 апреля 2023 г., $17: 29: 39$ 


\title{
ОТОБРАЖЕНИЯ И ПРЕОБРАЗОВАНИЯ ПОВОРОТА
}

\author{
(c) 2020 г. \\ Л. РЫПАРОВА, Й. МИКЕШ
}

\begin{abstract}
АннотАция. В статье изложены некоторые результаты, полученные для отображений и преобразований поворота. Здесь также приводится новые уравнения изопериметрических экстремалей поворота. Устранен ошибочный результат С. Г. Лейко, полученный для отображений поворота.
\end{abstract}

Ключевые слова: отображение поворота, преобразование поворота, изопериметрическая экстремаль поворота.

\section{ROTATION MAPPINGS AND ROTATION TRANSFORMATIONS}

\author{
(c) 2020 L. RÝPAROVÁ, J. MIKEŠ
}

\begin{abstract}
In this paper, we present some results obtained for rotation mappings and rotation transformations. We also obtain new equations for isoperimetric rotation extremals. Moreover, we propose a correction of an erroneous result of S. G. Leiko for rotation mappings.
\end{abstract}

Keywords and phrases: rotation mapping, rotation transformation, isoperimetric rotation extremal. AMS Subject Classification: 53B05, 53A05

1. Введение. Диффеоморфизмы поворота поверхностей $\mathcal{S}_{2}$ в трехмерном евклидовом пространстве $\mathbb{E}_{3}$, а также двумерных римановых многообразий $\mathbb{V}_{2}$ изучались в работах С. Г Лейко $[1-3,5,6,8-10,14]$. Эти результаты являются локальными и основаны на известном факте, что двумерное риманово многообразие $\mathbb{V}_{2}$ локально реализуется как поверхность $\mathcal{S}_{2}$ в $\mathbb{E}_{3}$.

Работа посвящена изложению результатов, полученных авторами в теории отображений поворота и преобразований поворота. Мы предлагаем более короткие решения по сравнению с указанными выше результатами Лейко. Кроме того, что в работе Лейко [1] нами обнаружена ошибка, которая, к сожалению, имеет влияние на последующие исследования.

Так как изучение отображений и преобразований поворота является предметом внутренней геометрии, ограничимся исследованием двумерного риманова пространства, при этом не исключаем псевдоримановы пространства $\mathbb{V}_{2}$.

2. Изопериметрические экстремали поворота. В этом разделе мы приводим результаты существования и единственности изопериметрических экстремалей поворота на двумерных (псевдо)римановых многообразиях $\mathbb{V}_{2}$ и на поверхностях $\mathcal{S}_{2}$ в евклидовом пространстве.

Изопериметрическая экстремаль поворота представляет собой кривую, определяемую следующим образом (см. [1]).

Работа выполнена при поддержке гранта Университета им. Ф. Палацкого (Оломоуц, Чехия) «Алгебраические и геометрические структуры», IGA PrF 2019015. 
Определение 1. Кривую $\ell$ называют изопериметрической экстремалъю поворота, если $\ell$ является экстремальной для $\theta[\ell]$ и $s[\ell]=$ const с фиксированными концами, где

$$
s[\ell]=\int_{t_{0}}^{t_{1}}|\lambda| d t, \quad \theta[\ell]=\int_{t_{0}}^{t_{1}} k(t) d t,
$$

$k$ и $\lambda$-кривизна и касательный вектор кривой соответственно.

С. Г. Лейко доказал (см. [1]), что в (неплоском) пространстве $\mathbb{V}_{2}$ кривая является изопериметрической экстремалью вращения тогда и только тогда, когда ее кривизна Френе $k_{g}$ и гауссова кривизна $K$ пропорциональны:

$$
k_{g}=c \cdot K,
$$

где $c=$ const. Также было доказано, что условие $k_{g}=c \cdot K$ можно записать в виде следующих уравнений внутреннего характера:

$$
\lambda_{2}=-\frac{g\left(\lambda_{1}, \lambda_{1}\right)}{g(\lambda, \lambda)} \cdot \lambda+\frac{\nabla_{\alpha} K \cdot \lambda^{\alpha}}{K} \cdot \lambda_{1},
$$

где $g\left(\lambda, \lambda_{1}\right)=0, \nabla_{i} K$ - градиентный вектор гауссовой кривизны $(K \neq 0)$ и

$$
\lambda_{2}^{h}=\nabla_{t} \lambda_{1}^{h} \equiv \frac{d \lambda_{1}^{h}}{d t}+\lambda_{1}^{\alpha} \Gamma_{\alpha \beta}^{h}(x(t)) \lambda^{\beta} .
$$

Эти уравнения требуют, чтобы метрика пространства принадлежала классу $C^{3}$, а единственность гарантирована при $C^{4}$.

В [16] эти уравнения упрощены так, что применимы для метрик класса дифференцируемости $C^{2}$. Имеет место следующая теорема.

Теорема 1. Уравнение изопериметрической экстремали поворота можно записать в виде

$$
\nabla_{s} \lambda=c \cdot K \cdot F \lambda, \quad c=\text { const. }
$$

Структура $F$ в $\mathbb{V}_{2}$ определена формулами

$$
F_{i}^{h}=g^{h \alpha} \varepsilon_{\alpha i}, \quad \varepsilon_{i j}=\sqrt{\left|g_{11} g_{22}-g_{12}^{2}\right|} \cdot\left(\begin{array}{cc}
0 & 1 \\
-1 & 0
\end{array}\right) ;
$$

она удовлетворяет условиям $F^{2}= \pm \mathrm{Id}$ и $\nabla F=0$.

Уравнения (3) проще, чем уравнения (2). Они имеют решение для метрик класса $C^{2}$.

Дальнейшим анализом приведенного выше уравнения были даны условия единственности изопериметрических экстремалей поворота.

Теорема 2 (см. [16]). Пусть $\mathbb{V}_{2}$ - (не плоское) риманово многообразие класса $C^{3}$. Тогда существует точно одна изопериметрическая экстремаль поворота, проходящая через точку $x_{0} \in \mathbb{V}_{2}$ в заданном неизотропном направлении $\lambda_{0} \in T \mathbb{V}_{2}$ для постоянной с.

3. Диффеоморфизм поворота. Определение диффеоморфизма поворота было введено в [1, 7] для поверхностей в евклидовом пространстве и двумерного собственно риманова многообразия $\mathbb{V}_{2}$.

Определение 2. Диффеоморфизм между двумерными римановыми многообразиями называется отображением поворота, если любая геодезическая отображается на изопериметрическую экстремаль поворота.

Понятие диффеоморфизма поворота обобщено в [12] следующим образом.

Пусть $\mathbb{V}_{2}=(M, g)$ - двумерное риманово многообразие $M$ с метрикой $g$ и $\overline{\mathbb{A}}_{2}=(\bar{M}, \bar{\nabla})-$ двумерное многообразие $\bar{M}$ с аффинной связностью $\bar{\nabla}$.

Определение 3. Диффеоморфизм $f: \mathbb{V}_{2} \rightarrow \overline{\mathbb{A}}_{2}$ называется отображением поворота, если любая геодезическая из $\overline{\mathbb{A}}_{2}$ отображается на изопериметрическую экстремаль поворота на $\mathbb{V}_{2}$. 
Дальнейшие исследования были сосредоточены на поиске условий, характеризующих отображения поворота. Доказана следующая теорема.

Теорема 3 (см. [12]). Пусть $\mathbb{V}_{2}$ допускает отображение поворота $f$ на $\overline{\mathbb{A}}_{2}$. Если $\mathbb{V}_{2}$ и $\overline{\mathbb{A}}_{2}$ в общей системе координат принадлежат классам $C^{2}$ и $C^{1}$ соответственно, то гауссова кривизна $K$ на $\mathbb{V}_{2}$ дифферениируема.

Из этой теоремы вытекает следующее утверждение.

Теорема 4. Если гауссова кривизна $K \notin C^{1}$, то диффеоморфизма поворота $\mathbb{V}_{2} \rightarrow \overline{\mathbb{A}}_{2}$ не существует.

Далее найдены фундаментальные уравнения диффеоморфизма поворота $\mathbb{V}_{2} \rightarrow \overline{\mathbb{A}}_{2}$, которые вытекают из определения 2 , когда $\mathbb{V}_{2} \in C^{2}$ и $\overline{\mathbb{A}}_{2} \in C^{1}$. Более того, теорема 3 налагает необходимое условие на гауссову кривизну: $K \in C^{1}$.

После более глубокого анализа (см. [12]) доказана следующая теорема.

Теорема 5. Риманово многообразие $\mathbb{V}_{2}$ допускает отображение поворота на $\overline{\mathbb{A}}_{2}$ тогда и только тогда, когда в $\mathbb{V}_{2}$ выполняется следующее уравнение:

$$
\nabla_{X} \theta=\left(\mathcal{A}(X)+\nabla_{X} K / K\right) \cdot \theta+\nu \cdot X
$$

для любого касательного вектора $X$, где $\nabla$-связность Леви-Чивиты, $K$ - гауссова кривизна, $\mathcal{A}$ - такая линейная форма, что $\mathcal{A}(X)=g(X, \theta), g$-метрический тензор, $\nu$ - некоторая функиия на $\mathbb{V}_{2}$.

С. Г. Лейко установил (см. [1]) уравнения (4) как необходимые условия существования отображения поворота двумерных римановых пространств. Эти условия (4) характеризуют $\theta$ как частный случай торсообразующего векторного поля.

В [1] (см. также $[2,5,8]$ ) Лейко утверждает, что из уравнений $(4)$ вытекает, что $\mathbb{V}_{2}$ изометрично поверхности вращения. В [11] доказано, что это утверждение неверно, но справедлива следующая теорема.

Теорема 6. Существует риманово пространство $\mathbb{V}_{2}$, которое не изометрично поверхности вращения, и в нем существует векторное поле, удовлетворяющее уравнениям (4).

Уравнения (4) можно записать в следующем виде:

$$
\theta_{j}^{h}=\theta^{h}\left(\theta_{j}+\partial_{j} \ln |K|\right)+\nu \delta_{j}^{h} .
$$

Очевидно, что $\theta$ является специальным торсообразующим векторным полем. Как известно, в любом $\mathbb{V}_{2}$ существуют торсообразующие векторные поля. В пространстве $\mathbb{V}_{2}$, метрика которого имеет вид

$$
d s^{2}=\left(d x^{1}\right)^{2}+f\left(x^{1}, x^{2}\right)\left(d x^{2}\right)^{2},
$$

где

$$
f=c\left(x^{2}\right) \cdot \exp \int F d x^{1}
$$

функция $F$ удовлетворяет следующему дифференциальному уравнению:

$$
\partial_{1} F=-\frac{F^{2}}{2}+\frac{F}{\varkappa}-2 \frac{\varkappa^{\prime}}{\varkappa^{2}} .
$$

Это уравнение - хорошо известное дифференциальное уравнение Бернулли относительно неизвестной функции $F$; переменная $x^{2}$ играет роль параметра. Общее решение этого уравнения зависит от одной произвольной функции параметра $x^{2}$, которая играет роль постоянной интегрирования. Поэтому не в каждом $\mathbb{V}_{2}$ существует вышеуказанное векторное поле. С другой стороны, имеет место следующая теорема. 
Теорема 7 (см. [17]). Двумерное (псевдо)риманово многообразие $\mathbb{V}_{2}$ допускает векторное поле поворота $\theta$ в том и только в том случае, когда в нем следующая замкнутая система типа Коши уравнений в ковариантных производных имеет решение относительно функиий $\theta_{i}(x)$ $u \nu(x)$ :

$$
\begin{aligned}
\theta_{i, j} & =\theta_{i}\left(\theta_{j}+\partial_{j} K / K\right)+\nu g_{i j}, \\
\nu_{i} & =\nu\left(\theta_{i}-\partial_{i} K / K\right)-K \theta_{i}-\theta_{\alpha} \theta_{\beta} g^{\alpha \beta} \partial_{i} K / K+\theta_{i} g^{\alpha \beta} \theta_{\alpha} \partial_{\beta} K / K .
\end{aligned}
$$

Для начальных условий Коши

$$
\theta_{i}\left(x_{0}\right)=\theta_{i}^{0}, \quad \nu\left(x_{0}\right)=\nu_{0},
$$

где $x_{0} \in \mathbb{V}_{2} \in C^{3}$, система (6) имеет не более одного решения $\theta_{i}(x), \nu(x)$. Также можно заменить условие $\mathbb{V}_{2} \in C^{3}$ более слабым условием: $g \in C^{2}$ и $K \in C^{1}$.

Отметим, что общее решение (6) для начальных условий (7) зависит не более чем от трех вещественных параметров, в качестве которых могут быть выбраны, например, числа $\theta_{i}^{0}$ и $\nu_{0}$.

4. Отображения поворота поверхностей вращения. Далее рассмотрим риманово многообразие, которое изометрично поверхности вращения. С. Г. Лейко (см. [1]) изучил уравнения отображений поворота поверхностей вращения и определил особые случаи поверхностей вращения, которые допускают эти отображения. Л. Рыпарова и Й. Микеш доказали (см. [19]), что любая поверхность вращения с дифференцируемой гауссовой кривизной допускает диффеоморфизм поворота, и то же самое верно для (псевдо)римановых пространств (эти результаты верны локально).

В статье Лейко [1] метрики поверхности вращения $\mathcal{S}_{2}$ представлены в виде

$$
d s^{2}=f(r) d r^{2}+r^{2} d \varphi^{2} .
$$

Он доказал, что отображение поворота римановых пространств $\mathbb{V}_{2}$ и $\overline{\mathbb{V}}_{2}$ существует тогда и только тогда, когда эти пространства является изометрическими поверхностям вращения, а метрики $\mathbb{V}_{2}$ и $\overline{\mathbb{V}}_{2}$ имеют следующую форму:

$$
\begin{aligned}
& \left(\tilde{g}_{i j}\right)=\frac{f(r)}{A^{2}(B+\sqrt{f(r)})^{2}} \operatorname{diag}\left(f(r), r^{2}\right), \\
& \left(\tilde{g}_{i j}\right)=B^{2} f(r) \operatorname{diag}\left(f(r), r^{2}\right), \quad A \neq 0, \quad B=\mathrm{const}
\end{aligned}
$$

(см. [1, теорема 2]).

Мы рассмотрели метрику поверхности вращения в более общем виде:

$$
d s^{2}=\left(d x^{1}\right)^{2}+f\left(x^{1}\right)\left(d x^{2}\right)^{2} .
$$

Эта метрика, в отличие от метрики (9), включает в себя горловые окружности.

Теорема 8 (см. [18]). Любая поверхность вращения $\mathcal{S}_{2}$ с дифферениируемой гауссовой кривизной $K$ допускает отображсение поворота на $\overline{\mathbb{A}}_{2}$.

Более того, эта теорема справедлива даже для псевдоримановых пространств, имеющих неопределенную метрику в случае, когда $f\left(x^{1}\right)<0$.

5. Бесконечно малые поворотные преобразования. Бесконечно малье преобразования (псевдо)римановых пространств $V_{n}$ можно в координатной форме определить следующим образом:

$$
\bar{x}^{h}=x^{h}+\varepsilon \xi^{h}(x),
$$

где $x^{h}$ - координаты определенной точки в $V_{n}$, и $\bar{x}^{h}$ являются координатами его изображения при бесконечно малом преобразовании, $\varepsilon$ является бесконечно малым параметром, не зависящим от $x^{h}$, и $\xi^{h}$ - вектор смещения (см. [13,15]).

В заключение в [19] доказана следующая теорема, которая значительно улучшает разультат [4]. 
Теорема 9. Дифференииальный оператор $\xi=\xi^{\alpha}(x) \partial_{\alpha}\left(\partial_{\alpha}=\partial / \partial x^{\alpha}\right)$ определяет бесконечно малое преобразование поворота (псевдо) риманова пространства $\mathbb{V}_{2}$ тогда и только тогда, когда $\xi$ удовлетворяет уравнениям

$$
\mathcal{L}_{\xi} \Gamma_{i j}^{h}=\delta_{(i}^{h} \psi_{j)}+\theta^{h} g_{i j}, \quad \theta_{i}^{h}=\theta^{h}\left(\theta_{i}+K_{i} / K\right)+\nu \delta_{i}^{h},
$$

где $\psi_{i}$-ковектор, $\delta_{i}^{h}$-символ Кронекера, $\theta^{h}$-векторное поле, $g$-метрический тензор, $K$ $(K \neq 0)$ - гауссова кривизна и $\mathcal{L}_{\xi}-$ производная Ли относительно $\xi$.

\section{СПИСОК ЛИТЕРАТУРЫ}

1. Лейко С. Г. Поворотные диффеоморфизмы на поверхностях евклидова пространства// Мат. заметки. - 1990. - 47, № 3. - C. 52-57.

2. Лейко С. Г. Вариационные задачи для функционалов поворота и спин-отображения псевдоримановых пространств// Изв. вузов. Мат. - 1990. - № 10. - С. 9-17.

3. Лейко С. Г. Экстремали функционалов поворота кривых псевдориманова пространства и траекторий спин-частиц в гравитационных полях// Докл. РАН. - 1992. - 325, № 4. - С. 659-663.

4. С. Г. Лейко Инфинитезимальные поворотные преобразования и деформации поверхностей евклидова пространства// Докл. РАН. - 1995. - 344, № 2. - С. 162-164.

5. Лейко С. Г. Изопериметрические экстремали поворота на поверхностях в евклидовом пространстве $E^{3} / /$ Изв. вузов. Мат. - 1996. - № 6. - С. 25-32.

6. Лейко С. Г. О конформных, конциркулярных и спиновых отображениях гравитационных полей// Мат. методы физ.-мех. поля. - 1997. - 40, № 2. - С. 44-47.

7. Лейко С. Г. Поворотные преобразования поверхностей// Мат. физ., анал., геом. $-1998 .-5$, № 3/4. - C. 203-211.

8. Лейко С. Г. Изопериметрические задачи для функционалов поворота первого и второго порядков в (псевдо)римановых многообразиях// Изв. вузов. Мат. - 2005. - № 5. - С. 49-55.

9. Лейко С. Г., Федченко Ю. С. Інфінітезимальні поворотні деформації поверхонь та їх застосування в теорії пружних оболонок// Укр. мат. ж. - 2003. - 55, № 12. - С. 1697-1703.

10. Лейко C. Г., аль Хуссен $C$. Поворотные квазиконциркулярные дифееоморфизмы на (псевдо)римановых пространствах// Мат. методы физ.-мех. поля. - 2001. - 44, № 1. - С. 22-27.

11. Микеш Й., Рыпарова Л., Худа Г. К теории поворотных отображений// Мат. заметки. - 2018. - 104, № 4. - С. $637-640$.

12. Chudá H., Mikeš J., Sochor M. Rotary diffeomorphism onto manifolds with affine connection// in: Geometry, Integrability, and Quantization. — Sofia: Bulgar. Acad. Sci., 2017. — Vol. 18. — P. 130-137.

13. Hinterleitner I. Special mappings of equidistant spaces// J. Appl. Math. - 2008. - 2. - P. 31-36.

14. Leiko $S$. G. Conservation laws for spin trajectories generated by isoperimetric extremals of rotation// Grav. Theor. Relativ. - 1988. - 26. - P. 117-124.

15. Mikeš J. et al. Differential Geometry of Special Mappings. - Olomouc, Czech Republic: Palacky Univ. Press, 2015.

16. Mikeš J., Sochor M., Stepanova E. On the existence of isoperimetric extremals of rotation and the fundamental equations of rotary diffeomorphisms// Filomat. — 2015. - 29, № 3. - P. 517-523.

17. Rýparová L., Kř́žek J., Mikešs J. On fundamental equations of rotary vector fields// Proc. 18th Conf. APLIMAT. - 2019. - P. 1030-1034.

18. Rýparová L., Mikeš J. On rotary mappings of surfaces of revolution// Proc. Int. Conf. "MITAV-2017" (Brno, June 15-16, 2017). — Brno, 2017. — P. 208-216.

19. Rýparová L., Mikeš J. Infinitesimal rotary transformation// Filomat — 2019. — 33, № 4.

Рыпарова Ленка

Университет им. Ф. Палацкого, Оломоуц, Чехия

E-mail: lenka.ryparova01@upol.cz

Микеш Йозеф

Университет им. Ф. Палацкого, Оломоуц, Чехия

E-mail: josef.mikes@upol.cz 